\title{
ON THE X-RAY HEATED SKIN OF ACCRETION DISKS
}

\author{
SERGEI NAYAKSHIN ${ }^{1}$ \\ NASA/GSFC, LHEA, Code 661, Greenbelt, MD, 20771 \\ Draft version July 30, 2021
}

\begin{abstract}
We present a simple analytical formula for the Thomson depth of the X-ray heated skin of accretion disks valid at any radius and for a broad range of spectral indices of the incident X-rays, accretion rates and black hole masses. We expect that this formula may find useful applications in studies of geometry of the inner part of accretion flows around compact objects, and in several other astrophysically important problems, such as the recently observed X-ray "Baldwin" effect (i.e., monotonic decrease of Fe line's equivalent width with the X-ray luminosity of AGN), the problem of missing Lyman edge in AGN, and line and continuum variability studies in accretion disks around compact objects. We compute the reflected X-ray spectra for several representative cases and show that for hard $\mathrm{X}$-ray spectra and large ionizing fluxes the skin represents a perfect mirror that does not produce any Fe lines or absorption features. At the same time, for soft X-ray spectra or small ionizing fluxes, the skin produces very strong ionized absorption edge and highly ionized Fe lines that should be observable in the reflected spectra.

Subject headings: accretion, accretion disks — radiative transfer — line: formation — X-rays: general — radiation mechanisms: non-thermal
\end{abstract}

\section{INTRODUCTION}

$\mathrm{X}$-ray illumination of an accretion disk surface is a problem of general astrophysical interest. Since the X-ray heating of the disk atmosphere changes energy and ionization balances there, the spectra emitted by X-ray illuminated accretion disks in any wavelength may be quite different from those resulting from non-illuminated disks. It has been known for many years (e.g., Basko, Sunyaev, \& Titarchuk 1974) that X-ray illumination leads to formation of a hot (and often completely ionized) X-ray "skin" above the illuminated material. Unfortunately, due to numerical difficulties, most previous studies of X-ray illumination had to rely on a constant density assumption for the illuminated gas (e.g., Ross \& Fabian 1993; Życki et al. 1994; Matt, Fabian \& Ross 1993; 1996; Ross, Fabian \& Brandt 1996), in which case the completely ionized skin forms only for very large ionization parameters (e.g., Ross, Fabian \& Young 1999).

Recently, Nayakshin, Kazanas \& Kallman (1999; hereafter paper I) have shown that if the assumption of the constant density is relaxed, then the temperature and ionization structure of the illuminated material is determined by the thermal ionization instability, and that the X-ray heated skin always forms on the top of the disk (see also Raymond 1993; Ko \& Kallman 1994; Różańska \& Czerny 1996). Due to the presence of the hot skin, the resulting reflected spectra are quite different from those obtained with the constant density assumption.

Unfortunately, computations similar to those reported in paper I are rather numerically involved and time consuming and thus are not readily performed. In this Letter, we present an approximate expression for the Thomson depth of the hot skin which allows one to qualitatively understand effects of the thermal instability on the reflected spectra. As an example, we apply our approximate expression to the observed X-ray Baldwin effect (e.g., Nandra et al. 1997b) in the geometry of a cold accretion disk illuminated by an X-ray source situated at some height above the black hole.

\footnotetext{
${ }^{1}$ National Research Council Associate
}

\section{THOMSON DEPTH OF THE SKIN}

We assume that X-rays are incident on the surface of an accretion disk whose structure is given by the standard accretion disk theory (e.g., SS73). Let us define the hot skin as the region of the gas where temperature significantly exceeds the effective temperature (see, e.g., Fig. 3b below). As explained in paper I, even $\mathrm{Fe}$ atoms are completely ionized in the X-ray skin if the $\mathrm{X}$-ray flux, $F_{\mathrm{x}}$, is comparable with or larger than the disk intrinsic flux, $F_{d}$, and if the illuminating $\mathrm{X}$-rays have relatively hard spectra, i.e., photon index $\Gamma \lesssim 2$. Under those conditions, the Compton temperature, $T_{c}$, is close to $\sim$ few keV. Therefore, we can neglect all the atomic processes and only consider Compton scattering and bremsstrahlung emission in our analytical study of the X-ray skin.

The Thomson depth of the hot layer, $\tau_{1}$, is obtained by integrating $d \tau_{1}=\sigma_{T} n_{e}(z) d z$, from $z=z_{b}$ to infinity, where $n_{e}(z)$ is the electron density; $z$ is the vertical coordinate; $z_{b} \simeq H$ gives the location of the bottom of the ionized skin; and $H$ is the disk scale height. Note that a simpler estimate of $\tau_{1}$ can be obtained if one assumes that the gas temperature is equal to the Compton one, and that the density law follows a Gaussian law (see Kallman \& White 1989).

In our calculations of $\tau_{1}$, we will neglect by the reprocessing of the radiation as it penetrates through the skin. This limits the applicability of our results to $\tau_{1} \lesssim 1$ if the angle, $\theta$, that the Xray radiation makes with the normal to the disk, is not too large, i.e., that $\mu_{i} \equiv \cos \theta$ is not too small. In the case when $\mu_{i} \ll 1$, the spectral reprocessing cannot be neglected for $\tau_{1} \gtrsim \mu_{i}$. For simplicity, we will assume $\mu_{i} \lesssim 1$ and postpone the treatment of large incident angles to a future publication.

If $P_{\text {bot }}$ is the gas pressure at $z=z_{b}$, then we can define dimensionless gas pressure as $P_{*} \equiv P / P_{\text {bot }}$, where $P=\rho / \mu k T$ and $\rho$ is the gas density, and also dimensionless gas temperature by $T_{*} \equiv T / T_{c}$ ( $T_{c}$ is the Compton temperature, see below). The Compton-heated skin then has $P_{*}<1$, whereas the colder material below the skin has $P_{*}>1$. As shown in paper $\mathrm{I}$, the incident $\mathrm{X}$-rays do not affect the hydrostatic balance in 
the ionized skin because the main source of opacity is Compton scattering (see Fig. 5b in paper I and also Sincell \& Krolik 1997). With this, one can re-write hydrostatic balance equation in terms of dimensionless variables as

$$
\frac{\partial P_{*}}{\partial x}=-2 \frac{P_{*}}{T_{*}} \frac{x-\zeta}{\lambda^{2}},
$$

where $x \equiv z / H, \zeta$ is the ratio of the disk midplane radiation pressure to the total pressure $\zeta=P_{\mathrm{rad}}(0) /\left(P_{\mathrm{rad}}(0)+\right.$ $\left.P_{\text {gas }}(0)\right)$, and $\lambda$ is the scale-height of the skin in units of $H$ : $\lambda^{2}=\left(2 k T_{c} R^{3} / G M \mu H^{2}\right)$. Expressing the electron density as $n_{e}=\rho / \mu_{e}$, one can arrive at

$$
\tau_{1}=\frac{\mu}{\mu_{e}} \frac{c P_{\mathrm{bot}}}{F_{\mathrm{x}}} \frac{l_{x}}{\theta_{c}} \lambda \int_{y_{b}}^{\infty} \frac{P_{*}}{T_{*}} d y \equiv \tau_{0} W\left(y_{b}\right),
$$

where $l_{x}$ is the compactness parameter of the illuminating $\mathrm{X}$ rays (see, e.g., equation 20 in paper I); $\theta_{c} \equiv k T_{c} / m_{e} c^{2}$; we also defined $y \equiv(x-\zeta) / \lambda$ and $y_{b}=\left(z_{b} / H-\zeta\right) / \lambda$; finally, $\tau_{0}$ is the expression before the sign of the integral. The integral in the equation (2) is designated as $W\left(y_{b}\right)$ and often (see below) turns out to be of order unity. Krolik, McKee \& Tarter (1981, $\S I V 2 b ;$ KMT hereafter) showed that for a completely ionized gas the energy balance equation can be written as

$$
T_{*}^{2}+T_{*}^{1 / 2} \Xi_{*}^{-1}-T_{*}=0,
$$

where $\Xi_{*} \equiv \Xi / \Xi_{i c}$ is the pressure ionization parameter normalized by the "inverse Compton" ionization parameter $\Xi_{i c}$, which is given by equation (4.5) of KMT, re-written in order to take into account the difference in definitions of $\Xi=F_{\mathrm{x}} / c P$ (the one used here) and $\Xi=F_{\text {ion }} / n_{H} k T$ (KMT; $F_{i o n}$ is the X-ray flux between 1 and $\left.10^{3} \mathrm{Ry}\right): \Xi_{i c}=0.47 * T_{8}^{-3 / 2} ; T_{8}$ here is the Compton temperature in units of $10^{8}$ Kelvin.

The solutions presented in paper I possess the property that the transition from the Compton-heated to cooler layers occurs at the upper bend of the S-curve (e.g., point (c) in Fig. 1 of paper I). In that point, $d T / d \Xi=\infty$. Using this condition, one can show that the transition happens at $T_{*}=1 / 3$ where $\Xi=\frac{3^{3 / 2}}{2} \Xi_{i c}$. Therefore,

$$
\begin{array}{r}
P_{\text {bot }}=\frac{2}{3 \sqrt{3}} \Xi_{i c}^{-1} \frac{F_{\mathrm{x}}}{c}=3.2 \times 10^{-2} T_{1}^{3 / 2} \frac{F_{\mathrm{x}}}{c} \\
\tau_{1} \simeq 6.9 T_{1} \frac{F_{\mathrm{x}}}{F_{d}} G^{1 / 2}(r) \dot{m}(1-f) \\
G(r) \equiv \frac{2^{16}}{27}\left[1-(3 / r)^{1 / 2}\right]^{2} r^{-3},
\end{array}
$$

where $T_{1}=k T_{c} / 1 \mathrm{keV} ; r=R / R_{s}, R_{s}=2 G M / c^{2}$ is the Schwarzschild radius; $\dot{m}$ is the dimensionless accretion rate ( $\dot{m}=1$ corresponds to Eddington luminosity for the accretion disk) and $0 \leq f<1$ is the coronal dissipation parameter (e.g., Svensson \& Zdziarski 1994). The maximum of function $G(r)$ occurs at $r=16 / 3$ where it is equal to unity. We also approximated $\mu_{e}=m_{p}, \mu=m_{p} / 2$, and $W\left(y_{b}\right) \simeq W(0)=1.23$ for the following reason. If the vertical pressure profile is given by $P(z)=P(0) \exp \left[-(z / H)^{2}\right]$ for the gas-dominated disk, the location of the temperature discontinuity is

$$
z_{b}=H \ln ^{1 / 2}\left[\frac{P(0)}{P_{\text {bot }}}\right]
$$

The function $\ln ^{1 / 2}(t)$ is a very slow, monotonically increasing one: for example, its value is 1.52 for $t=10$ and 3.72 for $t=10^{6}$. Therefore, for most realistic situations, $z_{b}$ is not very much larger than $H$. As is easy to check, $\lambda \gg 1$ for gas-dominated disks, and thus $y_{b} \ll 1$. For radiation-pressure dominated disks, $z_{b}$ is nearly equal to $H$ (see $\S 3.4$ in paper I), and hence $y_{b}=(1-\zeta) / \lambda \ll 1$ in this case as well. To summarize this statement in words, the vertical extent of the ionized skin is always large enough that the exact location of the inner boundary is unimportant, for either gas or radiation-dominated disks.

A cautionary note is in place. So far we assumed that the spectrum of the ionizing radiation does not change with the depth into the illuminated material. This is true only for optically thin situations, i.e., when $\tau_{1} \ll 1$, but for larger optical depths the Compton temperature is smaller than the one corresponding to $\tau_{1}=0$. The decrease in the local value of $T_{1}$ dictates a corresponding decrease in the value of $P_{\mathrm{bot}}$ according to equation (4), and therefore the transition to the cold equilibrium solution will always happen at a smaller $\tau_{1}$ than equation (5) predicts. To reflect this fact, Kallman \& White (1989) introduced $\tau_{\text {Th }}$ crit $\sim 1-10$, defined to be the maximum of $\tau_{1}$. Note that the behavior of $\tau_{\mathrm{h}}$ with Compton temperature, X-ray flux and radius given by equation (5) coincides with that obtained by these authors $\left(T_{\mathrm{IC} 8}\right.$ on the second line of their equation [1] should be in power +1 rather than $-1 / 2$ [T. Kallman 1999, private communication]).

With the understanding that for $\tau_{1} \gtrsim 1$ our analysis may not be expected to be very accurate, we choose to fix $\tau_{\text {Th }}$ crit at a value of 3 . This number is motivated only by the convenience - in paper I, due to the large volume of calculations, we have limited the Thomson depth of the illuminated gas that can be strongly ionized to $\tau_{T}=3$. This choice did not lead to any spurious results because the reprocessing features (from the cold material below the skin) become negligible already at $\tau_{T}=2$ (see paper I). In this paper, we use the following simple modification to the Thomson depth of the hot skin:

$$
\tau_{\mathrm{h}}=\frac{\tau_{1}}{1+\tau_{1} / 3},
$$

We can now compare our results with values of $\tau_{\mathrm{h}}$ numerically calculated in paper I. There, we conducted two types of tests. For radiation-dominated disks, we used fully selfconsistent formalism and thus we can directly compare results of $\S 5$ in paper I to results of equations (5) and (7). For purposes of isolating dependence of the reflected spectra on one parameter at a time and yet covering a large parameter space, we also conducted several tests where we artificially varied the "gravity parameter $A$ " (see $\S 4$ in paper I). In order to compare results of these latter tests, one should use the same approach that led us to equation ( 5 ), and also use the same gravity law as used in paper I with (note that in our current formulation, $\left.A=4 \theta_{c} l_{x}^{-1} \lambda^{-2}\right)$. This yields $\tau_{1}=0.73 T_{1}\left(l_{x} / A\right)^{1 / 2}$. Further, since $\lambda$ is now expressed as $\lambda^{2}=4 \theta_{c} A^{-1} l_{x}^{-1}$, the value of $y_{b}$ is not necessarily small, and thus $W\left(y_{b}\right)$ needs to be evaluated exactly. Finally, to locate the position of the lower boundary of the illuminated layer (which occurs almost at the same height as the temperature discontinuity - see Fig. $5 \mathrm{~d}$ in paper I for an example), we must substitute $P_{\text {bot }}$ in equation (6) on the value of gas pressure at Thomson depth $\tau_{\max }=4$, as used in paper I, which is approximately equal to $\tau_{\max } A F_{\mathrm{x}} / c=4 A F_{\mathrm{x}} / c$. 
The results of such a comparison are summarized in Figure (1), where we show $\tau_{\mathrm{h}}$ obtained in this paper versus that obtained numerically in paper I. The value of the Compton temperature (i.e., $T_{1}$ in eq. 5 ) is taken to be the temperature of the first zone in the temperature profiles shown in the corresponding Figures in paper I. We note that the deviation of our approximate expression from "exact" results is less than $\sim 20 \%$ even though we covered a wide range of physical conditions, i.e., strong to weak illumination limits, different indices of the incident X-ray radiation, and the disk itself is either gas or radiation dominated.

\section{3. "LAMP POST" MODEL}

As an application of our methods, we choose to analyze the model where the X-ray source is located at some height $h_{x}$ above the black hole (the "lamp post model" hereafter). Iwasawa et al. (1996) reported observations of iron line variability in Seyfert Galaxy MCG-6-30-15, and pointed out several problems connected with theoretical interpretation of these observations. Reynolds \& Begelman (1999) showed that the accretion flow within the innermost stable radius may be optically thick and thus produce fluorescent iron line emission in addition to such emission from the disk itself. These authors argued that the line emission from within the innermost stable radius may be important for the interpretations of the observations of Iwasawa et al. (see also Dabrowski et al. 1997). Reynolds et al. (1999) studied response of the iron line profiles to changes in the X-ray flux (iron line reverberation). In this paper we will not discuss the region within the innermost stable orbit for the reason that the properties of the accretion flow there are not well constrained. In particular, the hydrostatic and energy balance equations do not necessarily apply since the hydrostatic and thermal time scales can be longer than the in-fall time.

We will assume a non-rotating black hole and that all the Xrays are produced within the central source. We also neglect all relativistic effects in the present treatment. The projected X-ray flux impinging on the disk is

$$
F_{\mathrm{x}}=\frac{L_{x} h_{x}}{4 \pi\left(R^{2}+h_{x}^{2}\right)^{3 / 2}}
$$

Let us define $\eta_{x}$ as the ratio of the total X-ray luminosity to the integrated disk luminosity of the source, i.e., $\eta_{x} \equiv L_{x} / L_{d}$. Using equation (5), one obtains

$$
\tau_{1} \simeq 27.2 \frac{h_{x}}{R_{s}} \eta_{x} T_{1}\left[1+\left(h_{x} / R\right)^{2}\right]^{-3 / 2} r^{-3 / 2} \dot{m}
$$

(one still has to use equation []] to get the final answer for $\tau_{\mathrm{h}}$ ). For illustration, we choose a value of $h_{x}=6 R_{s}$. For $\Gamma=1.9$, a typical value for Seyfert Galaxies, the Compton temperature $k T_{x} \simeq 7.6 \mathrm{keV}$. As discussed in paper I (\$4.6), the Compton temperature at the surface of the disk depends on the cosine of the X-ray incidence angle, $\mu_{i}$, and the ratio $F_{\mathrm{x}} / F_{\mathrm{d}}$ approximately as:

$$
T_{c} \simeq T_{x}\left[1+\mu_{i} \sqrt{3} \frac{F_{\mathrm{x}}+F_{\mathrm{d}}}{F_{\mathrm{x}}}\right]^{-1} .
$$

Figure (2) shows the Thomson depth of the skin as a function of radius for several values of $\dot{m}$. The parameters in Figure (2) are chosen to be: (a) $\Gamma=1.9, \eta_{x}=1$ ("X-ray strong" case); (b) $\Gamma=1.9, \eta_{x}=0.1$ ("X-ray weak" case), and (c) $\Gamma=2.3$, $\eta_{x}=1$. Figure (3) shows the angle-averaged reflected spectra and temperature profiles of the hot layer for the three cases just considered and the accretion rate equal to the Eddington value ( $\dot{m}=1$ ) for $r=10$. The details of numerical methods with which these spectra were obtained are described in paper I.

If Compton temperature $T_{c} \gtrsim 1$, the illuminated gas is nearly completely ionized, and the line is emitted almost exclusively from the material below the skin. For this reason, it turns out that local emissivity of the line is negligible when $\tau_{\mathrm{h}} \gtrsim 1$ (see paper I). Thus, the strength of the iron line will be decreasing with $\dot{m}$ for the X-ray strong case (Fig. 2a) when $L_{x} \gtrsim$ few percent of the Eddington value. The skin is thickest for smaller radii, and therefore the broad iron line component will decrease first. The narrow line component (emitted farther away from the black hole) will also decrease with X-ray luminosity, but considerably slower than the broad line. In addition, if some of the line comes from a putative distant obscuring torus (Krolik, Madau \& Życki 1994), or the disk has a concave geometry (Blackman 1999), then the narrow component will decrease even slower. Thus, our qualitative description of the behavior of the iron line EW with luminosity for X-ray strong sources is consistent with Figure (3) of Nandra et al. (1997b), suggesting an explanation to the X-ray Baldwin effect.

The X-ray weak case (Figs. 2b \& 3b) is different in two respects. Firstly, the Thomson depth of the hot skin is smaller at a given accretion rate compared with the X-ray strong case. Most importantly, however, the skin is not "that hot". Namely, the skin temperature is only $\sim 0.3 \mathrm{keV}$ in the inner disk, and some of the iron ions are not completely ionized. For that reason, it turns out that the iron line centroid energy is close to 6.7 $\mathrm{keV}$, and a very deep absorption edge appears. This edge is in fact much stronger than the one resulting from a neutral material. Hence it is possible that this relatively cold X-ray heated skin can be unambiguously detected in spectra of real AGN.

Similarly, the soft incident X-ray spectrum leads to a relatively cool skin because $T_{x} \sim 1 \mathrm{keV}$ only (see Fig. $2 \mathrm{c} \& 3 \mathrm{c}$ ). As in the case $\eta_{x}=0.1$, a strong absorption edge is observed. In addition, the $6.7 \mathrm{keV} \mathrm{Fe}$ line is stronger, with $\mathrm{EW}$ of $65 \mathrm{eV}$. Therefore, such skin may also be detectable if it exists.

Comparing the value of $\tau_{\mathrm{h}}$ resulting from analytical formulae with those seen in Fig. (3b), one notes that deviations are as large as $\sim 50 \%$. These relatively large deviations exemplify the fact that our equations (5) and (7) are good approximations only to cases with strong X-ray flux and hard incident spectra (i.e., $F_{\mathrm{x}} \gtrsim F_{d}$ and $\Gamma \lesssim 2$ ). When one of these two conditions is not satisfied (and it is the case for all three cases presented in Figs. $2 \& 3$ ) the Compton heated layer is cool enough for atomic processes to provide additional sources of heating and cooling beyond Compton and bremsstrahlung processes, so that the energy equation (3) is not obeyed. However, our results can still be used as an order of magnitude estimate of $\tau_{\mathrm{h}}$ in the latter cases.

\section{SUMMARY}

In this Letter, we derived an approximate expression for the Thomson depth of the hot completely ionized skin on the top of an accretion disk illuminated by X-rays. Our results are only weakly dependent on the a priori unknown $\alpha$-viscosity parameter (because it enters through the boundary conditions). This allows us to reduce the uncertainty in documenting predictions of different accretion theories with respect to the iron line profiles and the strength of the X-ray reflection hump. Using the 
"lamp post model" geometry as an example, we showed that an inner part of an accretion disk may have a Thomson-thick ionized skin. Under certain conditions $\left(F_{\mathrm{x}} \gtrsim F_{d}\right.$ and $\left.\Gamma \lesssim 2\right)$, the X-ray heated skin may act as a perfect mirror for photons with energies below $\sim 30 \mathrm{keV}$. The physical cause of this is that Compton scattering in the skin prevents X-rays from penetrating to the deep cold layers that are capable of producing fluorescent line emission as well as setting other marks of atomic physics.

We note that because the ionized skin is thickest in the inner disk, the observed absence or deficit of the relativistically broadened line and other reprocessing features in some systems (e.g., Życki , Done \& Smith 1997, 1998), which was interpreted as a possible evidence for a disruption of the cold disk for small radii, may also mean that the "cold" disk is still present up to the innermost radius, but the skin effectively shields it from the X-ray illuminating flux. It is interesting, however, that the presence of the skin becomes apparent in systems that have $F_{\mathrm{x}} \lesssim F_{d}$ or $\Gamma \gtrsim 2$, because the Fe atoms are not completely stripped of their electrons and thus produce strong ion- ized edges and lines. We believe these predictions should be testable observationally with current X-ray missions such as Chandra, Astro-E and XMM. Also note that for a patchy corona model of accretion disks (e.g., Haardt, Maraschi \& Ghisellini 1994), the Thomson depth of the hot skin is always larger than the one found here, since the ratio $F_{\mathrm{x}} / F_{d}$ is larger. Finally, the presence of the ionized skin is important not only for the X-rays, but for other wavelengths as well (e.g., in studies of Lyman edge of accretion disks, correlation of optical/UV light curves with $\mathrm{X}$-rays).

A shortcoming of this paper is that we neglected the changes in the ionizing continuum due to scattering in the skin, which, strictly speaking, limits the applicability of our results to optically thin situations $\tau_{\mathrm{h}} \lesssim 1$ and incident angles not too far from normal $^{2}$. We plan to address this issue in future.

The author acknowledges support from NAS/NRC Associateship and many useful discussions with D. Kazanas, T. Kallman and M. Bautista. The anonymous referee is thanked for several constructive suggestions.

\section{REFERENCES}

Basko, M.M., Sunyaev, R.A. \& Titarchuk, L.G. 1974, A\&A, 31, 249

Blackman, E.G. 1999, ApJ, 306, L25

Dabrowski, Y., Fabian, A.C., Iwasawa, K., Lasenby, A.N., \& Reynolds, C.S. 1997, MNRAS, 288, L11

Haardt F., Maraschi, L., \& Ghisellini, G. 1994, ApJ, 432, L95

Iwasawa, K., et al. 1996, MNRAS, 282, 1038

Kallman, T.R., \& White, N. E. 1989, ApJ, 341, 955

Ko, Y-K, \& Kallman, T.R. 1994, ApJ, 431, 273

Krolik, J.H., McKee, C.F., \& Tarter, C.B. 1981, ApJ, 249, 422

Krolik, J.H., Madau, P., \& Życki, P.T. 1994, ApJ, 420, L57

Matt, G., Fabian, A.C., \& Ross, R.R.1993, MNRAS, 262, 179

Matt, G., Fabian, A.C., \& Ross, R.R. 1996, MNRAS, 278, 111

Nandra, K., George, I.M., Mushotzky, R.F., Turner, T.J., \& Yaqoob, T. 1997a, ApJ, 477, 602

Nandra, K., George, I.M., Mushotzky, R.F., Turner, T.J., \& Yaqoob, T. 1997b, ApJ, 488, L91
Nayakshin, S Kazanas, D. \& Kallman, T. 1999, submitted to ApJ fastroph/9909359).

Raymond, J.C. 1993, ApJ, 412, 267

Reynolds, C.S., \& Begelman, M.C. 1999, ApJ, 488, 109

Reynolds, C.S., Young, A.J., Begelman, M.C., \& Fabian, A.C. 1999, ApJ, 514, 164

Ross, R.R., \& Fabian, A.C. 1993, MNRAS, 261, 74

Ross, R.R., Fabian, A.C., \& Brandt, W.N. 1996, MNRAS, 278, 1082

Ross, R.R., Fabian, A.C., \& Young, A.J. 1999, MNRAS, 306, 461

Różańska, A., \& Czerny, P.T. 1996, Acta Astron., 46, 233

Shakura, N.I., \& Sunyaev, R.A. 1973, A\&A, 24, 337

Sincell, M.W., \& Krolik, J.H. 1997, ApJ, 476, 605

Svensson, R. \& Zdziarski, A. A. 1994, ApJ, 436, 599

Życki , P.T., Krolik, J.H., Zdziarski, A.A., \& Kallman, T.R. 1994, ApJ, 437, 597

Życki , P.T., Done, C., \& Smith, D.A. 1997, ApJ, 488, L113

Życki , P.T., Done, C., \& Smith, D.A. 1998, ApJ, 496, L25

\footnotetext{
${ }^{2}$ A crude "fix" to the problem of small incident angles is to use $c I_{x}$, where $I_{x}$ is the X-ray intensity integrated over $\phi$ angle, in place of $F_{\mathrm{x}}$ in equation (5)
} 


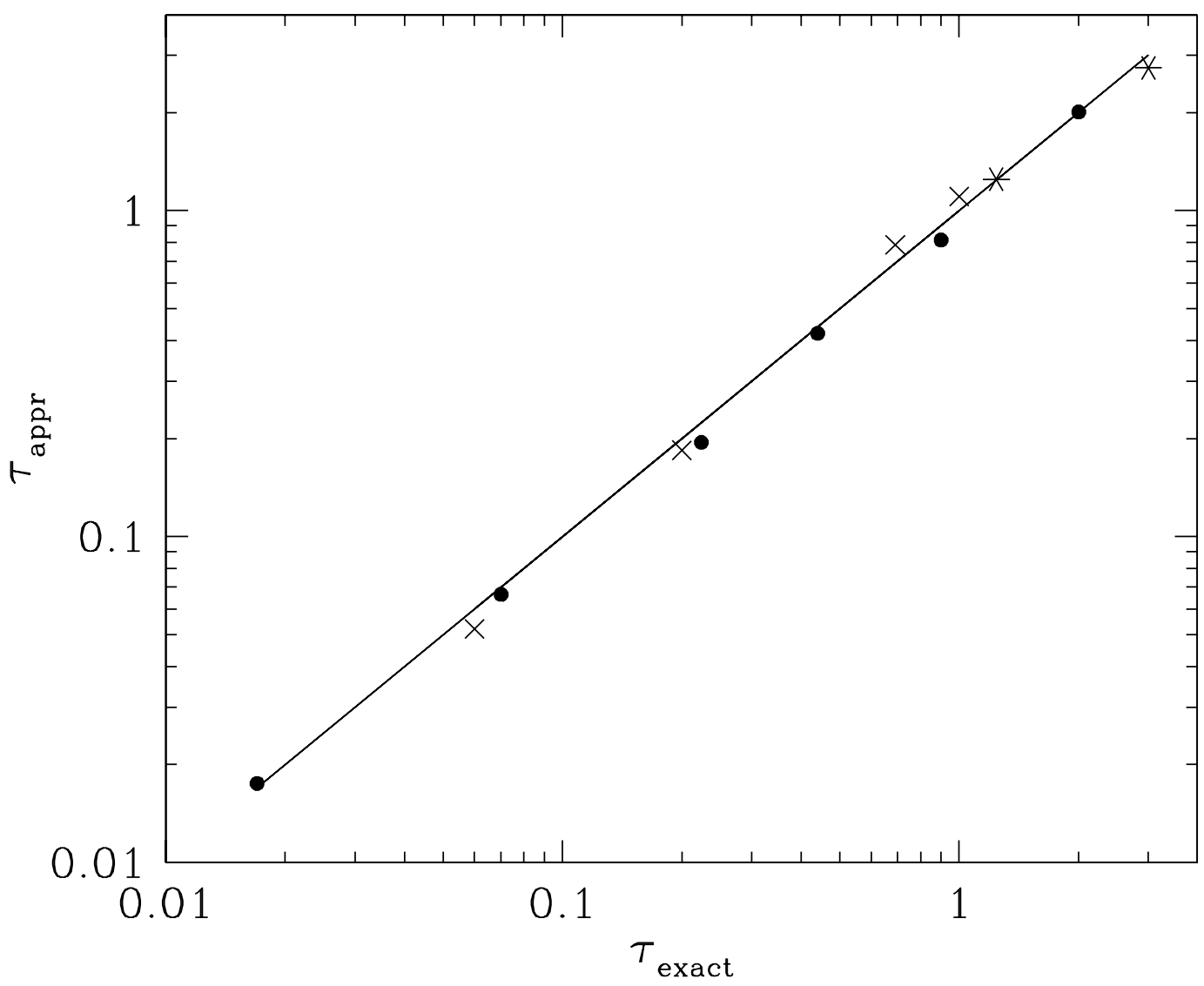

FIG. 1.- Comparison of the analytical approximation to the Thomson depth of the hot skin and the numerical results presented in paper I. Filled circles show tests with $\Gamma=1.9$, but with varying gravity parameter $A$ ( $\S \S 4.2-4.4$ in paper I); crosses show tests with $A=0.3$ and with $\Gamma$ varying between 1.5 and 2.3 ( $\S 4.7$ in paper I); and stars show the radiation-dominated case (described in $\S 5$ - dotted and long-dashed curves in Fig. 13 in paper I). The solid curve shows the line $\tau_{\text {approx }}=\tau_{\text {exact }}$. 


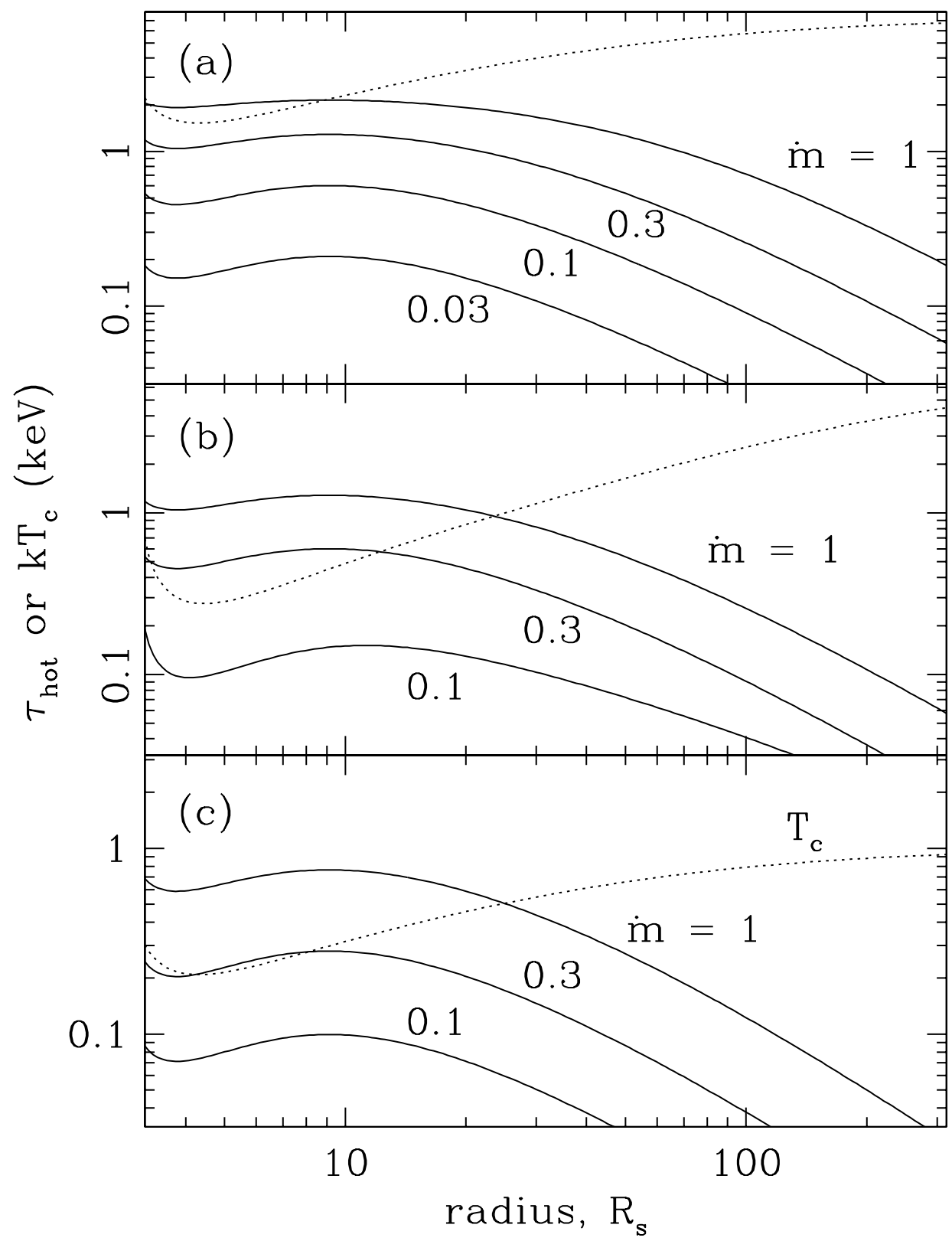

FIG. 2.- Thomson depth (solid curves) of the hot skin as a function of radius for different accretion rates (whose values are shown next to corresponding curves). The Compton temperature is shown by the dotted curve. See text for additional details. 

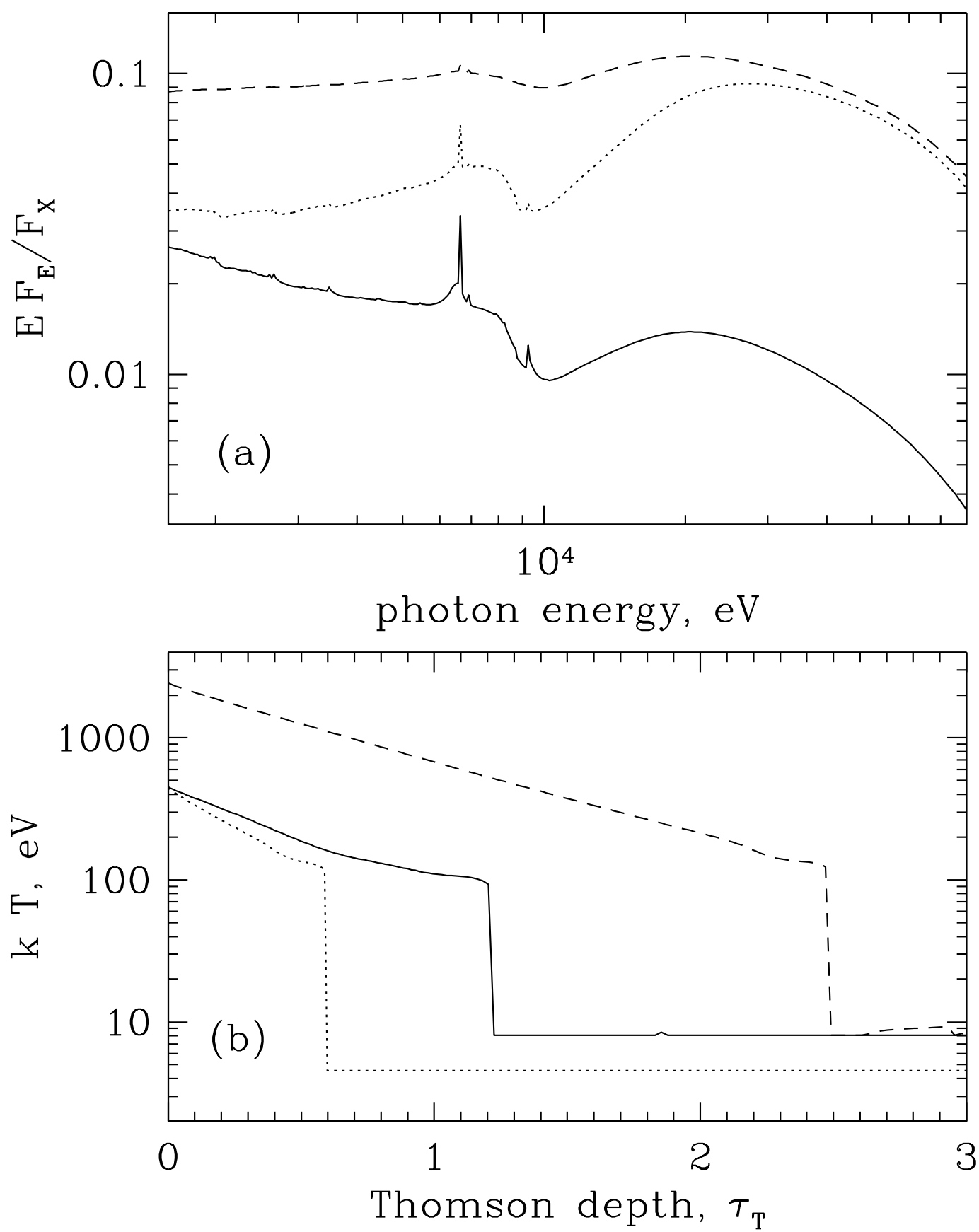

FIG. 3. - Reflected spectra (a) and temperature profile (b) computed for the lamp post geometry at $r=10$ with accretion rate $\dot{m}=1$. The dashed, dotted and solid curves correspond to models shown in Fig. (2) [a], [b] and [c], correspondingly. The solid curve in [a] was scaled down by factor of 2 for clarity. 